\title{
Effective pairing interactions with isospin density dependence
}

\author{
J. Margueron, ${ }^{1,2}$ H. Sagawa, ${ }^{2}$ and K. Hagino ${ }^{3}$ \\ 1 Institut de Physique Nucléaire, Université Paris-Sud, \\ $I_{2} P_{3}$-CNRS, F-91406 Orsay Cedex, France \\ ${ }^{2}$ Center for Mathematical Sciences, University of Aizu, \\ Aizu-Wakamatsu, 965-8580 Fukushima, Japan \\ ${ }^{3}$ Department of Physics, Tohoku University, Sendai, 980-8578, Japan
}

(Dated: November 17, 2018)

We perform Hartree-Fock-Bogoliubov (HFB) calculations for semi-magic Calcium, Nickel, Tin and Lead isotopes and $N=20,28,50$ and 82 isotones using densitydependent pairing interactions recently derived from a microscopic nucleon-nucleon interaction. These interactions have an isovector component so that the pairing gaps in symmetric and neutron matter are reproduced. Our calculations well account for the experimental data for the neutron number dependence of binding energy, two neutrons separation energy, and odd-even mass staggering of these isotopes. This result suggests that by introducing the isovector term in the pairing interaction, one can construct a global effective pairing interaction which is applicable to nuclei in a wide range of the nuclear chart. It is also shown with the local density approximation (LDA) that the pairing field deduced from the pairing gaps in infinite matter reproduces qualitatively well the pairing field for finite nuclei obtained with the HFB method.

PACS numbers: 21.30.Fe, 21.60.-n

Keywords: effective pairing interaction, isospin dependence, finite nuclei

\section{INTRODUCTION}

The origin of the pairing correlations in finite nuclei has been under debate since the formulation of the BCS theory [1] and its application to atomic nuclei [2, 3]. For many-electron systems, the phonon coupling is essential in order to get an attractive pairing interaction between electrons. In a marked contrast, the nuclear interaction is already attractive in the 
${ }^{1} \mathrm{~S}_{0}$ channel even without the phonon coupling. Nevertheless, it has been shown that the phonon coupling in uniform matter (often referred to as a medium polarization effect) as well as in finite nuclei may lead to an important contribution to the nuclear interaction in the particle-particle channel. In fact, several many-body methods have been developed recently for uniform matter in order to include these effects in the calculation of the pairing gap. These include a group renormalization method [4], Monte-Carlo calculations [5, 6, 7] and extensions of the Brueckner theory [8, 9]. Most of those calculations have been performed for pure neutron matter, because of the large interest in the application to neutron star physics. For instance, the pairing gap is important to understand the cooling of neutron stars, as it modifies the specific heat as well as some neutrino emission processes. These calculations, except for the one presented in Ref. [5], predict a reduction of the pairing gap in neutron matter.

It has been known that the pairing correlations play an important role in finite nuclear systems. The relation between finite nuclei and uniform matter, however, is not straight forward (see Ref. [10] for a complete review). In neutron stars, the number of protons is much smaller than that of neutrons. No finite nuclei have such extreme proton-to-neutron ratio. Also, the density ranges from very low densities up to several times nuclear matter saturation density in neutron stars, while it is close to the saturation density in finite nuclei.

Despite these differences, one might view finite nuclei as a point in the phase diagram, and extrapolate nuclear models to infinite matter under the extreme conditions realized in stars. Hence, there are mainly two different approaches for a calculation of pairing correlations in finite nuclei. The first approach is based on phenomenological pairing interactions whose parameters are determined using some selected data and the pairing interaction is usually not uniquely determined for the whole nuclear chart (see Refs. [11, 12] and references therein), while the second approach starts from a bare nucleon-nucleon interaction and eventually includes the effect of phonon coupling [13, 14, 15]. A calculation with the latter approach has recently been carried out, based on the nuclear field model. The results of this approach have suggested that the medium polarization effects significantly contribute to the pairing interaction in finite nuclei and in fact increase the pairing gap.

This result is apparently contradict with the results in infinite neutron matter, where the phonon coupling tends to reduce the pairing correlations. In order to understand the apparent contradiction, an extended Brueckner calculation including the medium polariza- 
tion effects has been performed in Ref. [9] both for symmetric and neutron matter. This calculation has shown that the medium polarization effects act differently in neutron matter and in symmetric matter. That is, the medium polarization effects do not reduce the pairing gap in symmetric matter, contrary to that in neutron matter. Instead, in symmetric matter, the neutron pairing gap is much enlarged at low density compared to that of the bare calculation without the polarization effect. This enhancement takes place especially for neutron Fermi momenta $k_{\mathrm{Fn}}<0.7 \mathrm{fm}^{-1}$. This provides at least a part of the reason why the medium polarization effects increase largely the pairing correlation in finite nuclei but decrease it in neutron matter.

In Ref. [16], we have proposed effective density-dependent pairing interactions which reproduce both the neutron-neutron $(n n)$ scattering length at zero density and the neutron pairing gap in uniform matter. In order to simultaneously describe the density dependence of the neutron pairing gap for both symmetric and neutron matter, it was necessary to include an isospin dependence in the effective pairing interaction. Depending on whether the medium polarization effects on the pairing gap given in Ref. [9] are taken into account or not, we have invented two different density dependences in the pairing interaction. The comparison of predictions of these interactions for finite nuclei with observed nuclear properties should shed light on the links between the origin of pairing in finite nuclei and that in uniform matter. This is the main motivation of this work, and, in this paper, we apply these interactions to semi-magic nuclei, such as $\mathrm{Ca}, \mathrm{Ni}, \mathrm{Sn}$ and $\mathrm{Pb}$ isotopic chains. We also investigate isotone chains such as $\mathrm{N}=20,28,50$, and 82 .

The paper is organized as follows. In Sec. II we briefly remind the main steps for the theoretical HFB approach and the procedure we have taken in Ref. [16] to construct the density-dependent contact pairing interactions. Results and predictions for the semi-magic $\mathrm{Ca}, \mathrm{Ni}, \mathrm{Sn}$ and $\mathrm{Pb}$ isotopes and $N=20,28,50$ and 82 isotones up to the expected drip lines are presented in Sec. III. In Sec. IV, a local density approximation is discussed in order to better understand the link with the uniform matter. Finally, the analysis of the results and the conclusions are given in Sec. $\nabla$. 


\section{HARTREE-FOCK-BOGOLIUBOV APPROACH WITH CONTACT DENSITY DEPENDENT INTERACTIONS}

The self-consistent Hartree-Fock-Bogoliubov (HFB) approach in coordinate representation has been presented in detail in Ref. [17]. For the sake of completeness, here we sketch briefly the main part of the model.

\section{A. HFB equations with spherical symmetry}

Assuming spherical symmetry and zero range effective nuclear interactions, the radial HFB equations have the form $(\tau=n, p)$ :

$$
\left(\begin{array}{cc}
\mathrm{h}_{\tau}(r)-\lambda_{\tau} & \Delta_{\tau}(r) \\
\Delta_{\tau}(r) & -\mathrm{h}_{\tau}(r)+\lambda_{\tau}
\end{array}\right)\left(\begin{array}{c}
\mathrm{U}_{\tau, i}(r) \\
\mathrm{V}_{\tau, i}(r)
\end{array}\right)=\mathrm{E}_{\tau, i}\left(\begin{array}{c}
\mathrm{U}_{\tau, i}(r) \\
\mathrm{V}_{\tau, i}(r)
\end{array}\right),
$$

where $\mathrm{E}_{\tau, i}$ is the quasiparticle energy, $\lambda_{\tau}$ is the chemical potential, $\mathrm{h}_{\tau}(r)$ is the mean field Hamiltonian, and $\Delta_{\tau}(r)$ is the pairing field. The HFB approach consists of solving Eq. (1) as a set of integrodifferential equations with respect to the amplitudes, $\mathrm{U}_{\tau, i}(r)$ and $\mathrm{V}_{\tau, i}(r)$, as functions of the position coordinate $r$. In the calculations presented here the mean field Hamiltonian $\mathrm{h}_{\tau}(r)$ is calculated with the SLy4 Skyrme force [18], and depends on the particle density,

$$
\rho_{\tau}(r)=\frac{1}{4 \pi} \sum_{i}\left(2 \mathrm{j}_{i}+1\right) \mathrm{V}_{\tau, i}^{*}(r) \mathrm{V}_{\tau, i}(r),
$$

as well as on the kinetic and the spin-orbit densities. In Eq. (2), the summation is done over the complete space, including bound and continuum states. For the pairing field, we use a density-dependent contact force as will be given in Eq. (5) in the next subsection. With this force the pairing field is local and is given by:

$$
\Delta_{\tau}(r)=\frac{\mathrm{v}_{0} \mathrm{~g}_{\tau}[\rho, \beta]}{2} \tilde{\rho}_{\tau}(r)
$$

where the total density is $\rho=\rho_{\mathrm{n}}+\rho_{\mathrm{p}}$, the asymmetry parameter is defined as $\beta=\left(\rho_{\mathrm{n}}-\rho_{\mathrm{p}}\right) / \rho$, and the pairing density $\tilde{\rho}_{\tau}(r)$ is

$$
\tilde{\rho}_{\tau}(r)=-\frac{1}{4 \pi} \sum_{i}\left(2 \mathrm{j}_{i}+1\right) \mathrm{U}_{\tau, i}^{*}(r) \mathrm{V}_{\tau, i}(r) .
$$

Because of the nature of the contact interaction, the pairing density $\tilde{\rho}_{\tau}$ is divergent, unless a cutoff energy is introduced in the sum $i$ of Eq. (4). 
The continuum states are modelized in this paper as discrete states provided by the finite-box boundary conditions $\left(R_{\mathrm{box}}=25 \mathrm{fm}\right)$. It has been proven that this approximation in the canonical basis provides an accurate description of the densities and the pairing densities [19, 20].

\section{B. The density-dependent pairing interactions}

In Ref. [16], we have taken a contact interaction $\mathrm{v}_{\tau \tau}$ acting on the singlet ${ }^{1} \mathrm{~S}$ channel,

$$
\left\langle k\left|\mathrm{v}_{\tau \tau}\right| k^{\prime}\right\rangle=\frac{1-\mathrm{P}_{\sigma}}{2} \mathrm{v}_{0} \mathrm{~g}_{\tau}[\rho, \beta] \theta\left(k, k^{\prime}\right),
$$

where the cutoff function $\theta\left(k, k^{\prime}\right)$ is introduced to remove the ultraviolet divergence in the particle-particle channel. A simple regularization could be done by introducing a cutoff momentum $k_{\mathrm{c}}$. That is, $\theta\left(k, k^{\prime}\right)=1$ if $k, k^{\prime}<k_{\mathrm{c}}$ and 0 otherwise. In finite systems, a cutoff energy $e_{\mathrm{c}}$ is usually introduced instead of a cutoff momentum $k_{\mathrm{c}}$. A detailed discussion on the different prescriptions for the cutoff energies in uniform matter are presented in Appendix A of Ref. [16]. For a sake of completeness of this paper, we report briefly the prescription 3 of Ref. [16] which is defined consistently with the HFB model. The cutoff is defined with respect to the quasi-particle energy $\sqrt{\left(\epsilon_{\mathrm{n}}(k)-\nu_{\mathrm{n}}\right)^{2}+\Delta_{\mathrm{n}}^{2}}<E_{\mathrm{c}}$. This leads to the following definition of the cutoff momenta:

$$
k_{\mathrm{c}}^{ \pm}=\left[2 \mathrm{~m}^{*}\left(\nu_{\mathrm{n}} \pm \sqrt{E_{\mathrm{c}}^{2}-\Delta_{\mathrm{n}}^{2}}\right)\right]^{1 / 2} / \hbar
$$

(if $E_{\mathrm{c}}>\Delta_{\mathrm{n}}$ ). If $k_{\mathrm{c}}^{-}$becomes imaginary for very small $\nu_{\mathrm{n}}$, we set $k_{\mathrm{c}}^{-}=0$. The parameters of the pairing interactions have been obtained within this prescription.

In Eq. (5), the interaction strength $\mathrm{v}_{0}$ is determined from the low-energy neutron-neutron scattering phase-shift [16, 21, 22, 23], that fixes the relation between $\mathrm{v}_{0}$ and the cutoff energy $e_{\mathrm{c}}$, while the density-dependent term $\mathrm{g}_{\tau}[\rho, \beta]$ is deduced from the realistic nucleonnucleon interaction calculations of the pairing gaps in symmetric and neutron matter. The isospin symmetry breaking of the bare nucleon nucleon interaction is neglected. The densitydependent term accounts for the medium effects and satisfies the boundary condition $\mathrm{g}_{\tau} \rightarrow 1$ for $\rho \rightarrow 0$. In Ref. [16], we have introduced an isovector dependence in the density-dependent term $\mathrm{g}_{\tau}[\rho, \beta]$ as $\mathrm{g}_{\tau}=\mathrm{g}_{\tau}^{1}+\mathrm{g}^{2}$. In the neutron pairing channel, the term $\mathrm{g}_{\mathrm{n}}^{1}$ is given as

$$
\mathrm{g}_{\mathrm{n}}^{1}[\rho, \beta]=1-\mathrm{f}_{\mathrm{s}}(\beta) \eta_{\mathrm{s}}\left(\frac{\rho}{\rho_{0}}\right)^{\alpha_{\mathrm{s}}}-\mathrm{f}_{\mathrm{n}}(\beta) \eta_{\mathrm{n}}\left(\frac{\rho}{\rho_{0}}\right)^{\alpha_{\mathrm{n}}},
$$




\begin{tabular}{ccccccc}
\hline \hline interaction & $E_{\mathrm{c}}$ & $\eta_{\mathrm{s}}$ & $\alpha_{\mathrm{s}}$ & $\eta_{\mathrm{n}}$ & $\alpha_{\mathrm{n}}$ & $\eta_{2}$ \\
\hline IS+IV Bare & $40 \mathrm{MeV}$ & 0.664 & 0.522 & 1.01 & 0.525 & 0.0 \\
IS+IV Induced & $40 \mathrm{MeV}$ & 1.80 & 0.27 & 1.61 & 0.122 & 0.8 \\
IS Bare & $40 \mathrm{MeV}$ & 0.664 & 0.522 & 0.664 & 0.522 & 0.0 \\
\hline \hline
\end{tabular}

TABLE I: Parameters for the density-dependent functions, $g_{1}$ and $g_{2}$ defined in Eqs. (7) and (8) ). These parameters are obtained from the fit to the pairing gaps in symmetric and neutron matter obtained by the microscopic nucleon-nucleon interaction. See the text for details.

where $\rho_{0}=0.16 \mathrm{fm}^{-3}$ is the saturation density of symmetric nuclear matter, and the term $\mathrm{g}^{2}$ is added only to the interaction IS+IV Induced and is given by

$$
\mathrm{g}^{2}[\rho]=\eta_{2}\left[\left(1+e^{\frac{k_{\mathrm{F}}-1.15}{0.05}}\right)^{-1}-\left(1+e^{\frac{k_{\mathrm{F}}-0.1}{0.08}}\right)^{-1}\right] .
$$

Notice the slight modifications of the parameters in Eq. 8 compare to [16]. In the proton pairing channel, the isospin symmetry of the matrix element (5) gives rise to the relation

$$
\mathrm{g}_{\mathrm{p}}^{1}[\rho, \beta]=\mathrm{g}_{\mathrm{n}}^{1}[\rho,-\beta] .
$$

The goal of the functional form in Eqs. (77) and (86) is to reproduce the theoretical calculation of the pairing gap in both symmetric and neutron matter and also to be used for prediction of the pairing gap in asymmetric matter. In finite nuclei, the densities $\rho_{\mathrm{n}}$ and $\rho_{\mathrm{p}}$ acquire an explicit dependence on the coordinate $r$, which defines the density $\rho(r)$ and the asymmetry parameter $\beta(r)$. In Eq. (17), the interpolation functions $\mathrm{f}_{\mathrm{s}}(\beta)$ and $\mathrm{f}_{\mathrm{n}}(\beta)$ should satisfy the following conditions, $\mathrm{f}_{\mathrm{s}}(0)=\mathrm{f}_{\mathrm{n}}(1)=1$ and $\mathrm{f}_{\mathrm{s}}(1)=\mathrm{f}_{\mathrm{n}}(0)=0$. It should however be noticed that the interpolation functions $\mathrm{f}_{\mathrm{s}}(\beta)$ and $\mathrm{f}_{\mathrm{n}}(\beta)$ cannot be deduced from the adjustment of the pairing gap in symmetric and neutron matter. In this paper, we choose $\mathrm{f}_{\mathrm{s}}(\beta)=1-\mathrm{f}_{\mathrm{n}}(\beta)$ and $\mathrm{f}_{\mathrm{n}}(\beta)=\beta$.

We adjust the parameters of the contact pairing interaction so that the position and the absolute value of the maxima of the pairing gaps of the nucleon-nucleon interaction in symmetric and neutron matter are reproduced. For the bare pairing gap, the maximum is located at $k_{\mathrm{Fn}}=0.87 \mathrm{fm}^{-1}$ with $\Delta_{\mathrm{n}}=3.1 \mathrm{MeV}$ for both symmetric and neutron matter, 
while for the screened pairing gap, the maximum is at $k_{\mathrm{Fn}}=0.60 \mathrm{fm}^{-1}$ with $\Delta_{\mathrm{n}}=2.70 \mathrm{MeV}$ for symmetric matter and $k_{\mathrm{Fn}}=0.83 \mathrm{fm}^{-1}$ and $\Delta_{\mathrm{n}}=1.76 \mathrm{MeV}$ for neutron matter. We call the interaction fitted to the bare pairing gap the IS+IV Bare interaction, while that to the screened gap the IS+IV Induced interaction. In order to estimate the importance of the isovector term of the interaction, we have also parameterized a pure isoscalar interaction, IS Bare, so as to reproduce the bare pairing gap in symmetric matter. The obtained

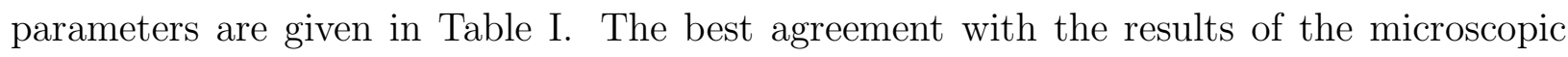
nucleon-nucleon interaction in Ref. [9] is obtained with a cutoff energy $E_{\mathrm{c}}=40 \mathrm{MeV}$ [16].

\section{RESULTS FOR FINITE NUCLEI}

It is a rather difficult task to extract the pairing gaps from the experimental data to compare with the theoretical results (see for instance Ref. [25] and references therein). In the following, we thus compare the predictions of the pairing interactions with different experimental data [26]: the masses per particle $\mathrm{B}(N, Z) / A$, two neutrons separation energies defined as $\mathrm{S}_{2 \mathrm{n}}=\mathrm{B}(N, Z)-\mathrm{B}(N-2, Z)$, and the odd-even mass staggering (OES) defined as

$$
\begin{aligned}
\Delta^{(3)}(N, Z) \equiv & -\frac{\pi_{N}}{2}[\mathrm{~B}(N-1, Z)-2 \mathrm{~B}(N, Z) \\
& +\mathrm{B}(N+1, Z)]
\end{aligned}
$$

where $\pi_{N}=(-)^{N}$ is the number parity. For even nuclei, the OES is known to be sensible not only to the pairing gap, but also to shell effects and deformations [25, 27]. Therefore, the comparison of a theoretical pairing gap with OES should be done with caution. At a shell closure, the OES (10) does not go to zero as expected, but it increases substantially (see Fig. 1). This large gap is an artifact due to the shell effect, which is totally independent of the pairing gap itself. In the following, we shall thus remove all the nuclei at the shell closures from the comparison to experimental OES.

The effects of the isospin asymmetry on the pairing gap has been suggested for a long time. In Ref. [28], the mass number dependence of the pairing gap has been extracted from the experimental OES for nuclei outside the shell closures within the range $50<Z<82$ and $82<N<126$. Two phenomenological fits have been suggested. The first one, which we call isoscalar, is only dependent on the mass number $A$, and reads $\Delta_{n}^{\mathrm{IS}}=13.3 / A^{1 / 2} \mathrm{MeV}$. On 
the other hand, the second one, which we call isovector, has a quadratic dependence on the neutron-proton asymmetry, and is expressed as $\Delta_{\mathrm{n}}^{\mathrm{IS}+\mathrm{IV}}=\left[7.2-44(1-2 Z / A)^{2}\right] / A^{1 / 3} \mathrm{MeV}$. We represent in Fig. 1 the experimental OES $\Delta_{\mathrm{n}}^{(3)}$ in Eq. (10) together with the phenomenological fits, $\Delta_{\mathrm{n}}^{\mathrm{IS}}$ and $\Delta_{\mathrm{n}}^{\mathrm{IS}+\mathrm{IV}}$. From comparisons between the fits and the experimental OES in Fig. 1, it is difficult to extract the quadratic dependence of the pairing gap. Namely, the fits $\Delta_{\mathrm{n}}^{\mathrm{IS}}$ and $\Delta_{\mathrm{n}}^{\mathrm{IS}+\mathrm{IV}}$ reproduce the experimental OES equally well in general, despite an appreciable difference in the predictions in very heavy isotopes. The fitting functions are supposed to describe the smooth behavior of the pairing gaps with $A$ and $Z$, but are not able to describe the fine structure of the pairing gap in a single nuclei. For instance, the drop of the pairing gap at a shell closure is totally absent.

The experimental binding energies per particle $\mathrm{B} / A$ is compared with our results for the two pairing interactions, IS+IV Bare and IS+IV Induced, in Fig. 2. The results can be classified into two groups: the first group is the one of the light isotopes ( $\mathrm{Ca}$ and $\mathrm{Ni}$ ) for which the HF calculation is already close to the experimental masses, while the second group is the one of the heavier isotopes $(\mathrm{Sn}$ and $\mathrm{Pb}$ ) for which $\mathrm{HF}$ calculations underestimate the binding energies (see the solid line). When the pairing is switched on, the interaction IS+IV Induced reproduces the experimental masses in the first group of isotopes within the

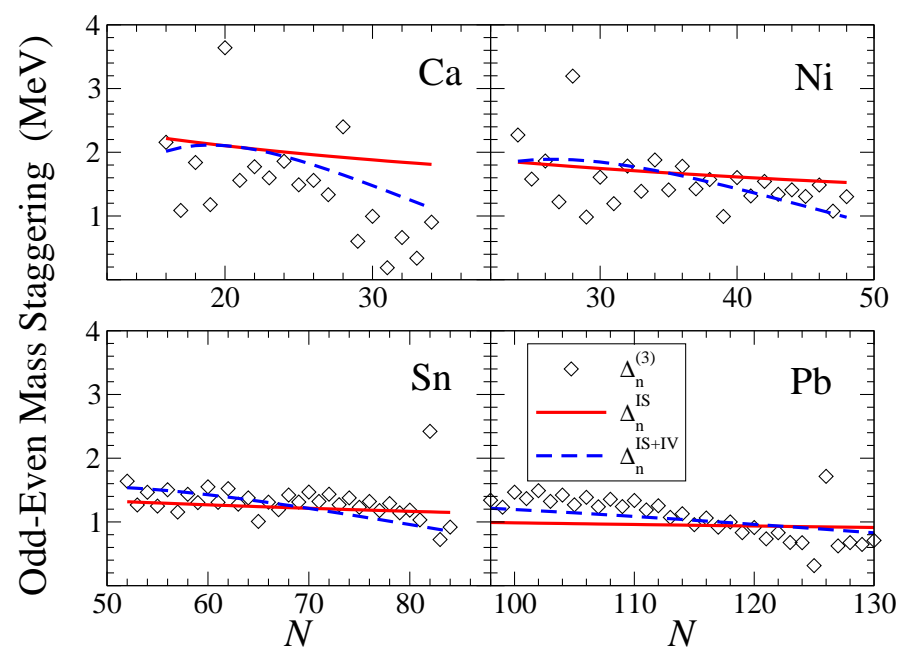

FIG. 1: (Color online) The experimental odd-even mass staggering $\Delta_{\mathrm{n}}^{(3)}$ given by Eq. (10) for the semi-magic $\mathrm{Ca}, \mathrm{Ni}, \mathrm{Sn}$ and $\mathrm{Pb}$ isotopes. It is compared with the phenomenological fits $\Delta_{\mathrm{n}}^{\mathrm{IS}}=$ $13.3 / A^{1 / 2} \mathrm{MeV}$ and $\Delta_{\mathrm{n}}^{\mathrm{IS}+\mathrm{IV}}=\left[7.2-44(1-2 Z / A)^{2}\right] / A^{1 / 3} \mathrm{MeV}$ proposed in Ref. [28]. 

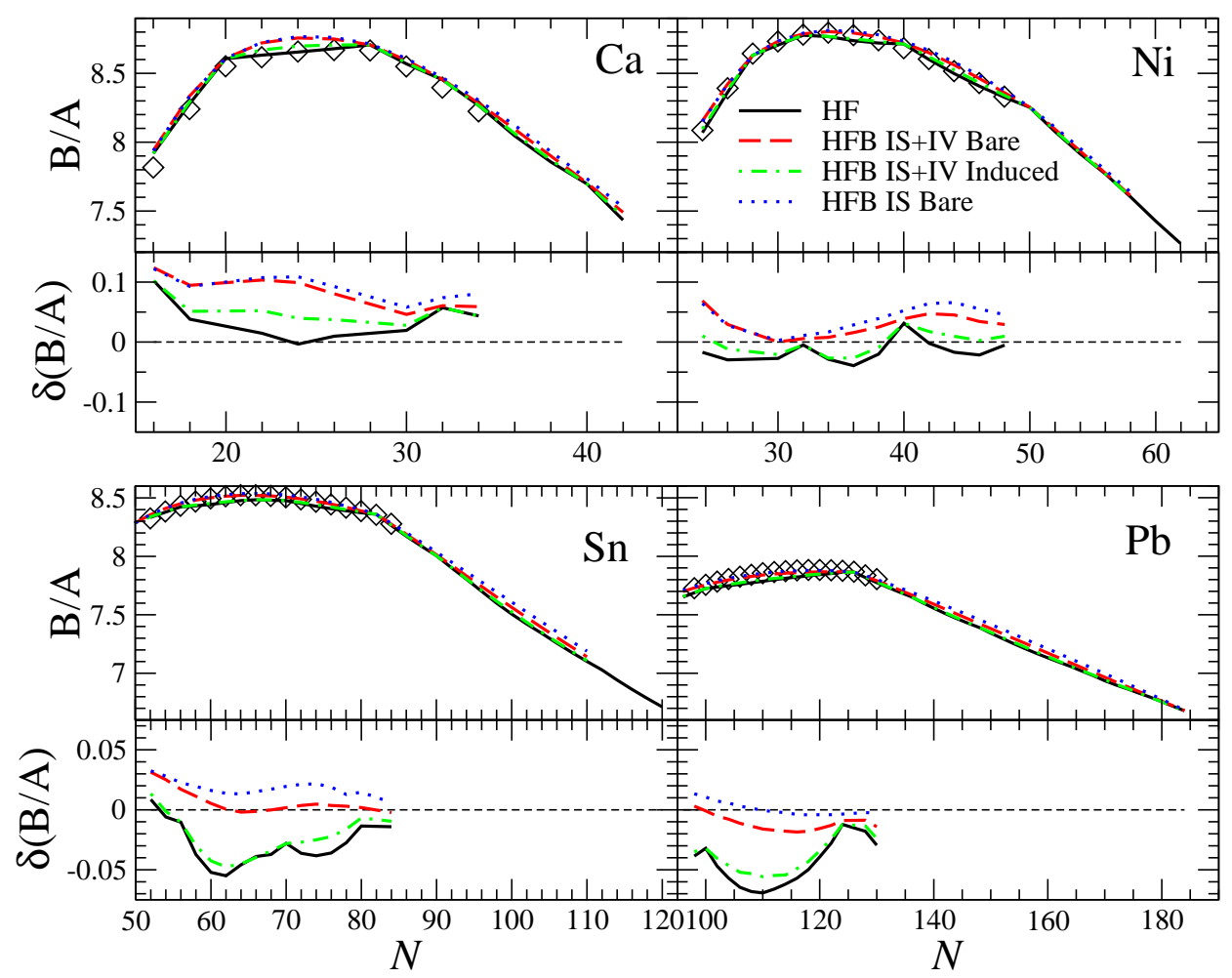

FIG. 2: (Color online) Comparison of the HFB calculations with the experimental binding energies, $\mathrm{B} / A$. The solid line shows the results without pairing interaction (HF), while the dotted, short dashed, and long dashed lines are obtained with the pairing interactions IS+IV Bare, IS+IV Induced and IS Bare, respectively. For each isotopic chain, we also plot the difference $\delta(\mathrm{B} / A)=\mathrm{B}($ th. $) / A-\mathrm{B}($ exp. $) / A$ between the theoretical and the exprimental values for the binding energy. All units are given in $\mathrm{MeV}$. See the text for more details.

same accuracy of HF, while the interaction IS+IV Bare overestimates the masses for this group. In contrast, the second group of isotopes behaves in an opposite way: the pairing interaction IS+IV Bare leads to masses which are much closer to the experimental ones as compared to the IS+IV Induced interaction, or to the HF calculation. The interaction IS Bare will be discussed latter on. When one compares the difference between the theoretical and the experimental binding energies $\delta(\mathrm{B} / A)$, it is observed that the pairing interaction IS+IV Bare flattens this difference as a function of neutron number, even for the first group of light isotopes. These results suggest that the different behavior between the first and the second groups, rather than a pairing effect, originates from an effect of the mean field Skyrme interaction (SLy4), which has been parameterized so as to reproduce better the 

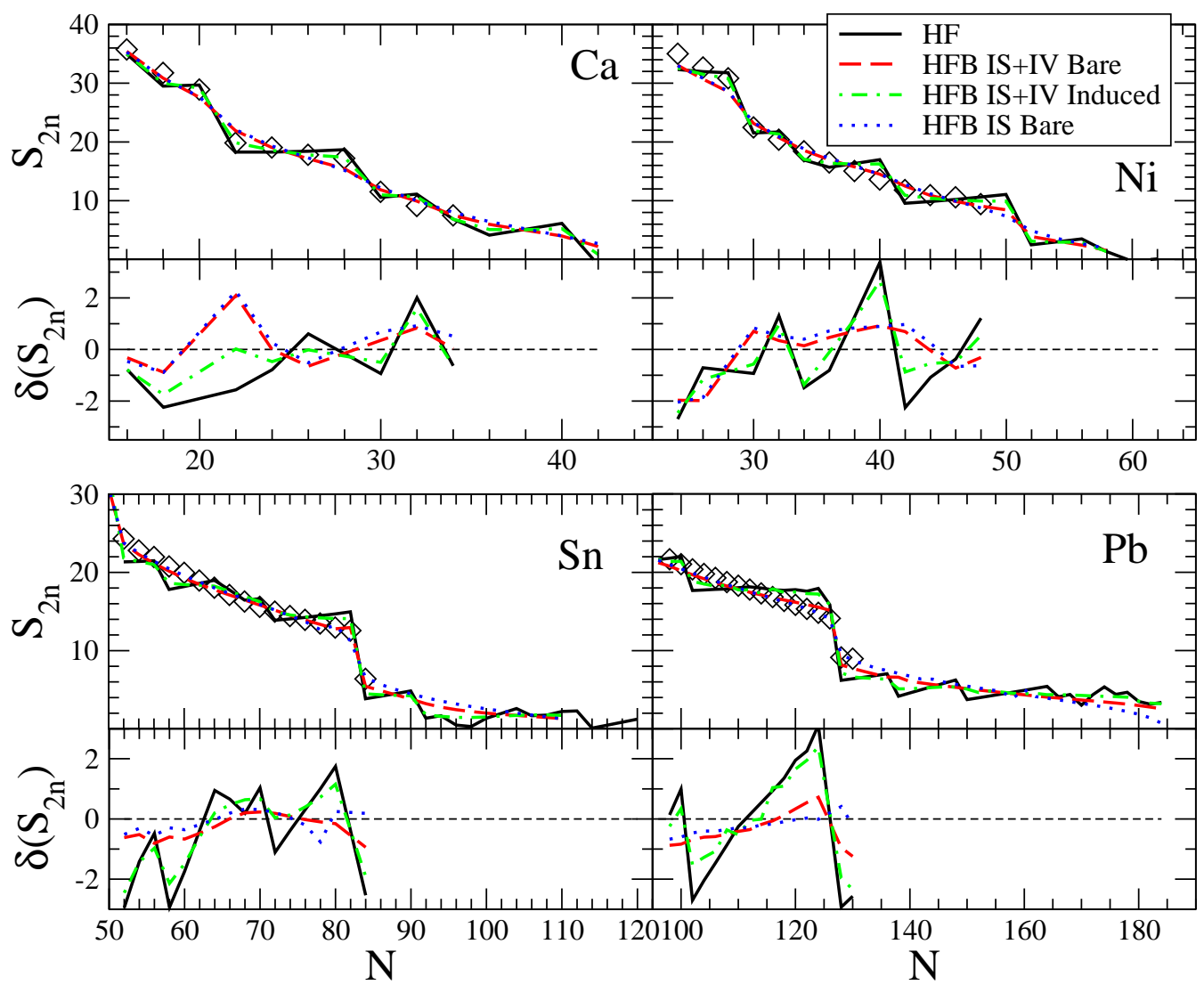

FIG. 3: (Color online) Comparison between HFB and experiments for the two-neutron separation energies $\mathrm{S}_{2 \mathrm{n}}$. The value $\delta\left(\mathrm{S}_{2 \mathrm{n}}\right)$ is defined as $\delta\left(\mathrm{S}_{2 \mathrm{n}}\right)=\mathrm{S}_{2 \mathrm{n}}($ th. $)-\mathrm{S}_{2 \mathrm{n}}$ (exp.). See the caption of Fig. 2 and the text for details.

binding energies of intermediate and heavy nuclei rather than those of the light ones.

The effect of the pairing correlations can be clearly seen in the two neutrons separation energy $\mathrm{S}_{2 \mathrm{n}}$, which is sensible to the relative difference in binding energies, and somehow reduces the effect of the mean field interaction. The results of $\mathrm{HFB}$ calculations for $\mathrm{S}_{2 \mathrm{n}}$ are shown in Fig. 3 in comparison with experimental data. We now see that the pairing interaction IS+IV Bare works better than HF or the IS+IV Induced interaction for all the four selected isotopic chains. The dependence of $\mathrm{S}_{2 \mathrm{n}}$ on the neutron number $N$ is much improved using the interaction IS+IV Bare than IS+IV Induced, even for the group of light isotopes. It should be reminded that no tuning for each isotopes has been done for any of these pairing interactions.

Let us next compare the experimental OES with the mean pairing gap calculated from 

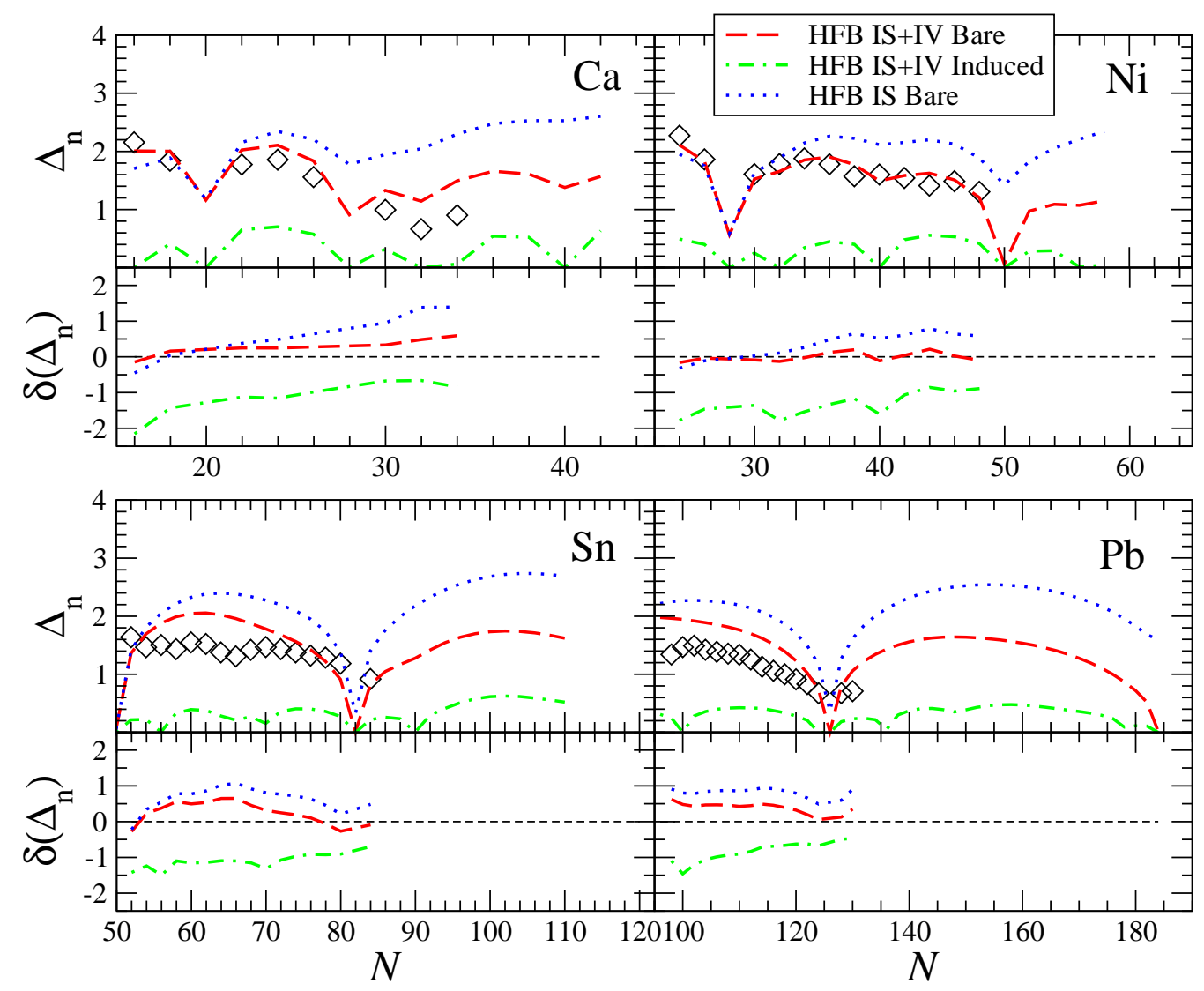

FIG. 4: (Color online) Comparison of the HFB pairing gaps $\Delta_{\mathrm{n}}$ calculated with Eq. (11) with the OES given by $\Delta^{(3)}$. The value $\delta\left(\Delta_{n}\right)$ is defined as $\delta\left(\Delta_{n}\right)=\Delta_{n}($ th. $)-\Delta_{n}$ (exp.). See the caption of Fig. 2 and the text for details.

the pairing field $\Delta_{\mathrm{n}}(r)$ as,

$$
\Delta_{\mathrm{n}} \equiv \frac{1}{N} \int d^{3} r \rho_{\mathrm{n}}(r) \Delta_{\mathrm{n}}(r),
$$

where $N=\int d^{3} r \rho_{\mathrm{n}}(r)$ is the number of neutrons. In the next section, we will discuss also another formula for the mean pairing gap. The results are shown in Fig. 4. We remind the reader that this comparison should be taken with caution and we have removed from the comparison the OES calculated at the neutron shell closure. It is observed that the pairing gaps obtained with the interaction IS+IV Induced are systematically too small along the isotopic chains. This is the reason why the results with the interaction IS+IV Induced are close to the HF calculations in Figs. 2 and 3. Contrary, the results with the interaction IS+IV Bare are in good agreement with the experimental OES, including the isotopic trend for all the four isotopic chain. 

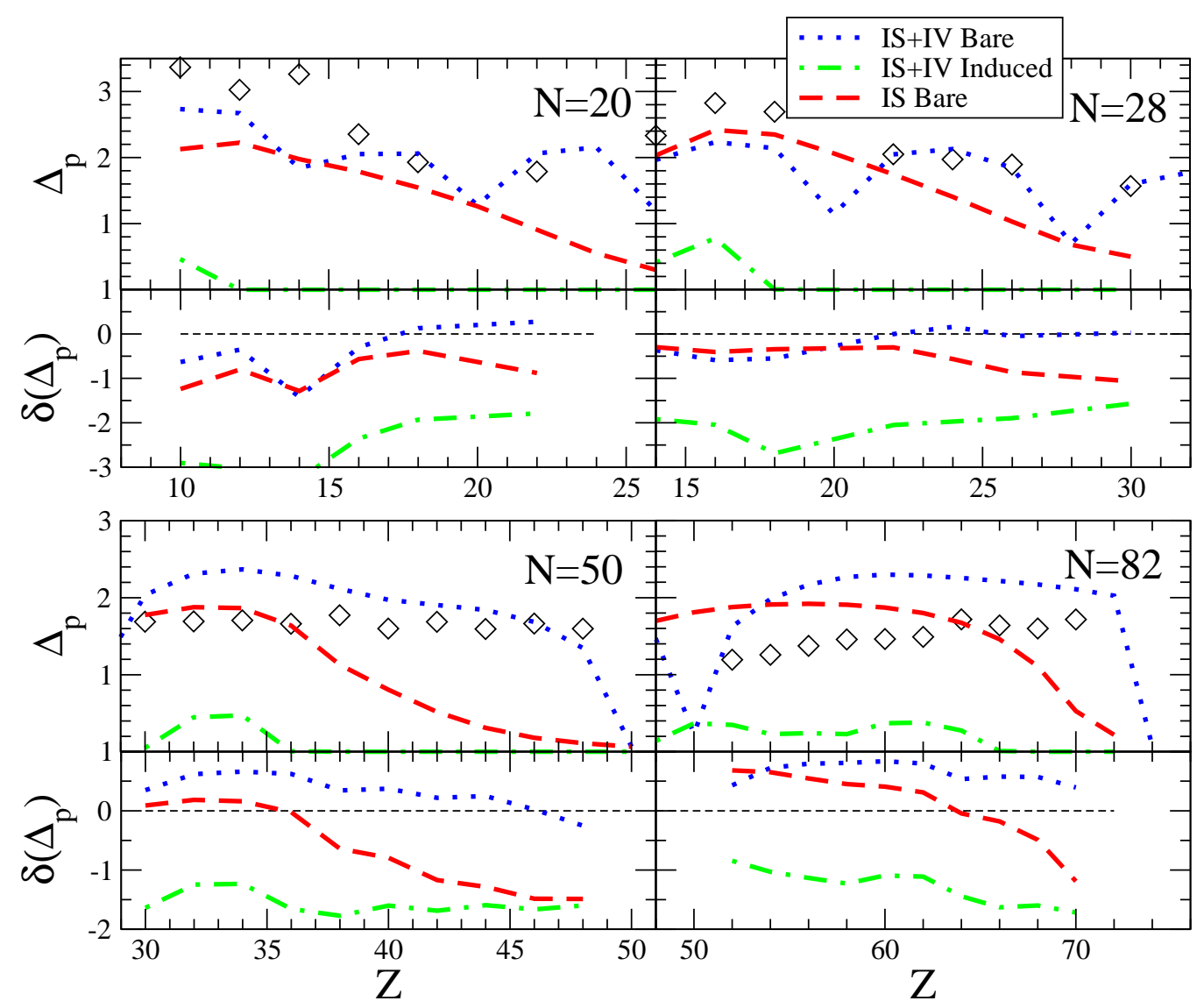

FIG. 5: (Color online) Comparison of the HFB pairing gaps $\Delta_{\text {p }}$ calculated with Eq. (11) with the OES given by $\Delta^{(3)}$. The value $\delta\left(\Delta_{\mathrm{p}}\right)$ is defined as $\delta\left(\Delta_{\mathrm{p}}\right)=\Delta_{\mathrm{p}}($ th. $)-\Delta_{\mathrm{p}}($ exp.). See the caption of Fig. 2 and the text for details.

The proton-proton pairing interaction should also be analyzed in order to design a global pairing interaction applicable in the whole nuclear chart, It should however be noticed that in our calculations the Coulomb interaction has not been included in the pairing channel. The effect of the Coulomb interaction on proton pairing gap has however been estimated for instance in Ref. [29] and is expected to decrease the pairing gap by 100 to $200 \mathrm{keV}$. A pertubative estimate of the Coulomb effect on the proton pairing gap has been evaluated and is expected to be of order of $0.5-1 \mathrm{MeV}$ on the pairing gain energy [30]. This is consistent with the estimation of Ref. [29] for the pairing gap. Neglecting the Coulomb effect, our calculation is therefore a semi-quantitative estimate of the proton pairing gap, which could still be interesting in order to analyze its isotonic dependence. In Fig. 5 we explore the proton pairing gap in some isotonic chains such as e.g. $N=20,28,50$ and 82 . The figure 
shows significant improvement in proton-rich isotones by IS+IV Bare pairing compared with IS Bare only. As already observed in the neutron channel, the IS+IV Induced pairing interaction is not strong enough to lead to reasonable proton pairing gaps.

In order to understand the differences between the interactions IS+IV Bare and IS+IV Induced, we plot in Fig. [6 the pairing gaps in symmetric, asymmetric (asymmetry parameter $\beta=0.4)$ and neutron matter obtained with these interactions. From this figure, it is clear that the pairing gap for symmetric matter obtained with the interaction IS+IV Induced is much smaller than that with the interaction IS+IV Bare for $k_{\mathrm{Fn}}>0.7 \mathrm{fm}^{-1}$, causing the weak pairing effects in the finite nuclei. The medium polarization effects estimated in Ref. [9] shift the density at the peak position of the pairing gap by a factor of $\sim 3$ from that of the bare gap, i.e., $k_{\mathrm{Fn}} \sim 0.87 \mathrm{fm}^{-1}\left(\rho_{\mathrm{n}} \sim 0.22 \times 10^{-3} \mathrm{fm}^{-3}\right)$ to $k_{\mathrm{Fn}} \sim 0.60 \mathrm{fm}^{-1}$ $\left(\rho_{\mathrm{n}} \sim 0.73 \times 10^{-4} \mathrm{fm}^{-3}\right)$. This change may cause an enhancement of the pairing correlations in very low density regime and cause BCS-BEC crossover phenomena [16]. However, the comparison with the experimental OES shown in Fig. 4 clearly indicates that this medium polarization effect estimated in infinite matter gives rise to too weak pairing correlations in finite nuclei.

Let us now discuss the role of the isovector term. Since the pairing interaction IS+IV Bare is in good agreement with the experimental data as shown in Figs. 2, 3 and 4, we focus on this pairing interaction. To this end, we construct an isoscalar pairing interaction, IS Bare, which is fitted to the bare gap as for the interaction IS+IV Bare, but using only the gap in the symmetric nuclear matter. The parameters for the interaction IS Bare is listed in Table I (notice $\eta_{\mathrm{s}}=\eta_{\mathrm{n}}$ and $\alpha_{\mathrm{s}}=\alpha_{\mathrm{n}}$ ). The pairing gap in uniform matter obtained with this isoscalar interaction is shown in Fig. 6 by the dashed line. While in symmetric matter, the interactions IS+IV Bare and IS Bare lead to identical pairing gaps to each other, the isoscalar interaction IS Bare produces a much larger pairing gap than the IS+IV Bare interaction, as the asymmetry increases. Moreover, the parameters $\eta_{\mathrm{s}}$ and $\eta_{\mathrm{n}}$ in Table $\llbracket$ show that the IS+IV Bare interaction is of mixed surface and volume type in symmetric matter $\left(\eta_{\mathrm{s}}=0.664\right)$ as suggested in Ref. [31], and of pure surface type in neutron matter $\left(\eta_{\mathrm{n}}=1.01\right)$, while the IS Bare pairing interaction is of mixed type independently of the asymmetry. This difference should manifest itself in the results of finite nuclei. The binding energy, the two neutrons separation energy, and the average pairing gap obtained with the interaction IS Bare are shown by the dashed line in Figs. 2, 3 and 4, respectively. It is clearly seen that while these 


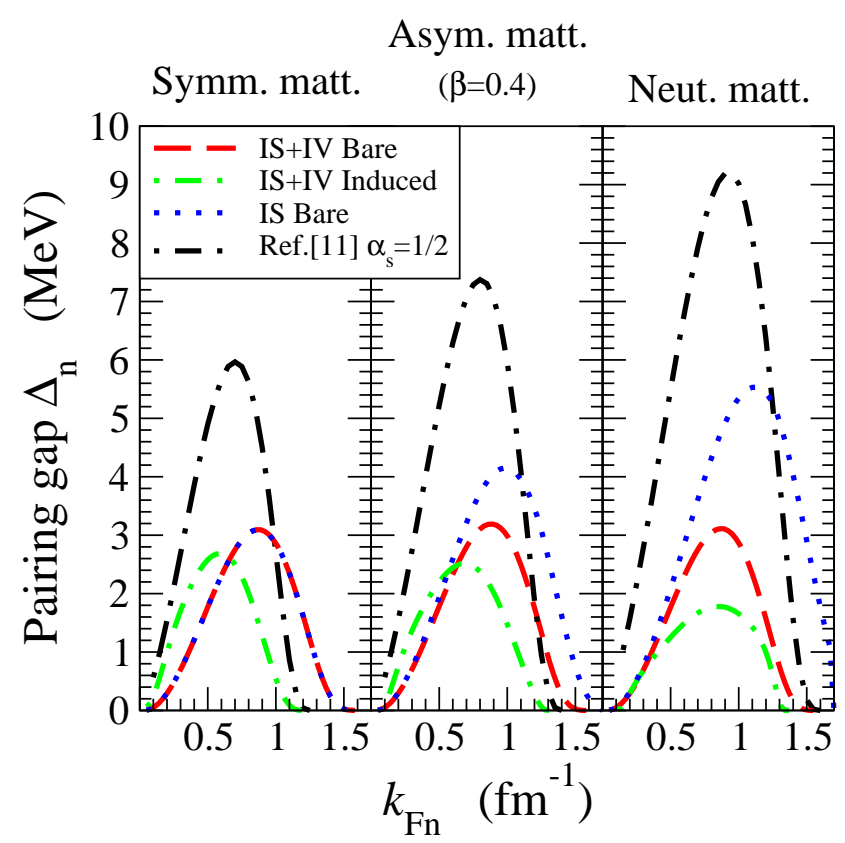

FIG. 6: (Color online) Pairing gaps in uniform matter obtained from the solution of the BCS equations with the pairing interactions IS+IV Bare, IS+IV Induced, IS Bare and the isoscalar interaction of Ref. [11] with $\alpha_{\mathrm{s}}=1 / 2$ and $\eta_{\mathrm{s}}=1$. Notice that the results of IS+IV Bare is identical to those of IS Bare in symmetric matter. See the text for more details.

interactions produce similar results for $N=Z$ nuclei, the isotopic behavior is somewhat different. From Fig 4 , it is seen that both the interactions IS+IV Bare and IS Bare produce arches of the paring gap in between the neutron magic numbers, but the arches induced by the isoscalar pairing interaction IS Bare are much larger in amplitude than the one produced by the interaction IS+IV Bare. The difference between the calculated and the experimental pairing gap, $\delta\left(\Delta_{\mathrm{n}}\right)$, estimated with the interaction IS+IV Bare is indeed flatter than that with the interaction IS Bare. This behavior suggests clearly the importance of the isovector component of the pairing interactions as it has already been shown for uniform matter in Fig. 6. We believe that this will bring an important improvement in the description of pairing in nuclei. Further information of the isovector pairing interaction might be obtained from experimental study of binding energies of very exotic nuclei and excitation spectra of various isotopes.

It was pointed out that lower power of the density dependence $\alpha_{\mathrm{s}}<1 / 2$ with $\eta_{\mathrm{s}}=1$ in the isoscalar pairing interaction gives rise to anomalous behavior in the particle and 
pairing densities in neutron rich nucleus ${ }^{150} \mathrm{Sn}$ [11]. In Fig. 7, the asymptotic behaviour of the particle and pairing densities obtained for the set of interactions in Ref. [11] are compared with these obtained for the IS+IV Bare interaction in this neutron rich nucleus. We represent the densities only for the IS+IV Bare interaction because in the asymptotic tail, the IS+IV Bare, IS+IV Induced and IS Bare are almost undistinguishable. It is shown that despite the fact that the value of the power of the density dependence is around $1 / 2$ for the IS+IV Bare and IS Bare interactions, and less for the IS+IV Induced interaction, no anomalous behavior in the densities is observed, contrary to the interactions studied in Ref. [11]. We have represented the pairing gaps in symmetric, asymmetric and neutron matter for the isoscalar interaction with $\alpha_{\mathrm{s}}=1 / 2$ and $\eta_{\mathrm{s}}=1$ in Fig. 6] (see the dot-dashed line). This interaction induces large values of the pairing gaps at low density from symmetric to neutron matter. We have indeed found that the interactions with power of the density dependence $\alpha_{\mathrm{s}}=1 / 2,1 / 3$ and $1 / 6$ in Ref. [11] have a peak in the pairing gap of absolute value of about $6 \mathrm{MeV}$ at $k_{\mathrm{Fn}} \sim 0.7 \mathrm{fm}^{-1}\left(\rho_{\mathrm{n}} \sim 10^{-2} \mathrm{fm}^{-3}\right)$ in symmetric nuclear matter. The pairing gaps are even increasing when going from symmetric to neutron matter, as we already pointed out as a typical behavior for isoscalar pairing interactions. Hence, the anomalous behavior described in Ref. [11] might be related to an anomalous value of the pairing gap at very low density rather than to the value of the power of the density dependence of the pairing interaction, as it was claimed. In Ref. [11], the pure surface interactions with $\eta_{\mathrm{s}}=1$ have been adjusted to the value of the pairing gap $\Delta_{\mathrm{n}}=1.25 \mathrm{MeV}$ in ${ }^{120} \mathrm{Sn}$. From our study, one could conclude that these pure surface interactions do not reproduce the pairing gaps in uniform matter obtained from the bare microscopic nucleon nucleon interaction. In order to reproduce them, it is indeed necessary to take the parameter $\eta_{\mathrm{s}}$ as adjustable and generate mixed surface and volume pairing interactions, as it has been done in Refs. [16, 23, 31].

The interaction IS+IV Bare can be parameterized in a form

$$
\begin{aligned}
& \mathrm{g}_{\mathrm{n}}^{3}\left[\rho_{\mathrm{n}}, \rho_{\mathrm{p}}\right]=1-\eta_{1}\left(\frac{\rho_{\mathrm{n}}}{\rho_{0}}\right)^{\alpha_{1}}-\eta_{2}\left(\frac{\rho_{\mathrm{p}}}{\rho_{0}}\right)^{\alpha_{2}}, \\
& \mathrm{~g}_{\mathrm{p}}^{3}\left[\rho_{\mathrm{n}}, \rho_{\mathrm{p}}\right]=1-\eta_{1}\left(\frac{\rho_{\mathrm{p}}}{\rho_{0}}\right)^{\alpha_{1}}-\eta_{2}\left(\frac{\rho_{\mathrm{n}}}{\rho_{0}}\right)^{\alpha_{2}},
\end{aligned}
$$

with the parameters $\eta_{1}=1.01, \alpha_{1}=0.525, \eta_{2}=-0.058, \alpha_{2}=0.559$, and the cutoff energy $E_{\mathrm{c}}=40 \mathrm{MeV}$. The bare pairing gap could be reproduced by setting $\mathrm{g}_{\tau}=\mathrm{g}_{\tau}^{3}$ in Eq. (5). In the neutron pairing channel, the very weak dependence on the proton density is shown from the value of the parameter $\eta_{2}$ which is close to zero. With the density-dependent terms (12) and 


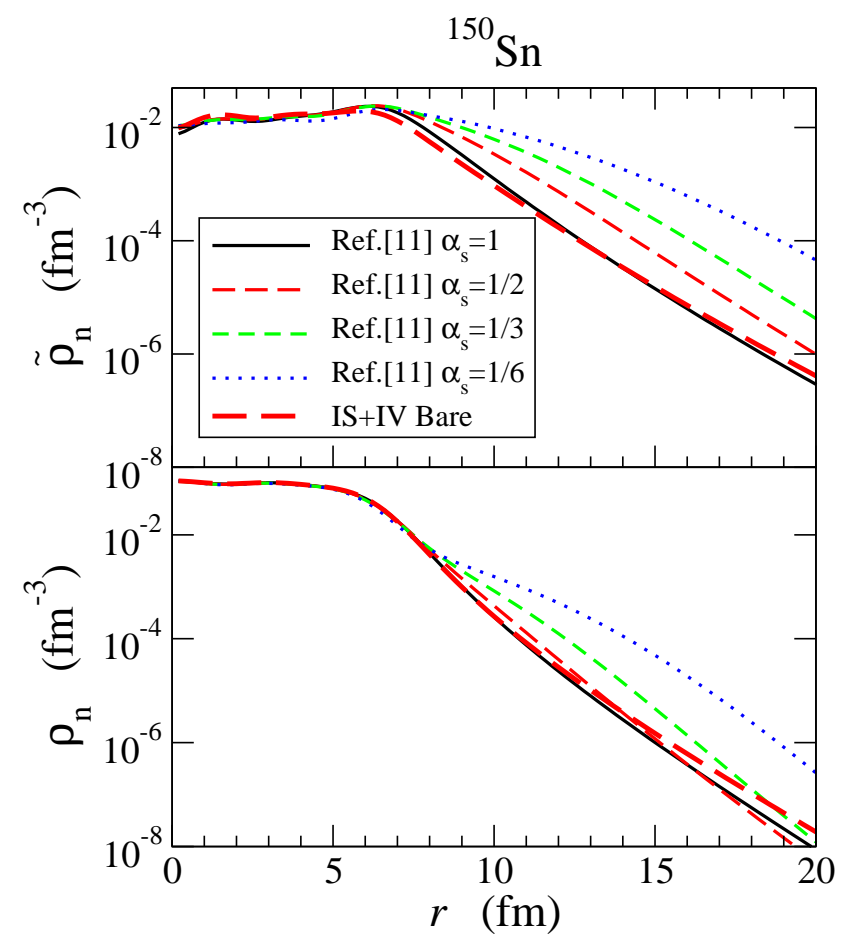

FIG. 7: (Color online) Comparision of particle and pairing densities for the nucleus ${ }^{150} \mathrm{Sn}$ obtained with several sets of interaction in Ref. [11] and with the IS+IV Bare interaction. See the text for more details.

(13), we can obtain similar results in finite nuclei to the ones obtained with the interaction IS+IV Bare with the terms (7) and (9).

Let us discuss the qualitative relation between the density-dependent term $\mathrm{g}_{\tau}^{1}$ and $\mathrm{g}_{\tau}^{3}$ in the case of the IS+IV Bare interaction. Neglecting $\eta_{2}$ and expressing the variables as $\rho_{\mathrm{n}}=(1+\beta) \rho / 2$ and $\rho_{\mathrm{p}}=(1-\beta) \rho / 2$ in Eqs. (12) and (13), we obtain to the first order in $\beta$ that $\mathrm{g}_{\tau}^{3} \approx \mathrm{g}_{\tau}^{1}$ if the following relations are respected: $\eta_{\mathrm{s}}=\eta_{1} / 2^{\alpha_{1}}, \alpha_{\mathrm{s}}=\alpha_{1}, \eta_{\mathrm{n}}=$ $\eta_{\mathrm{s}}\left(1+\alpha_{1}\right)$, and $\alpha_{\mathrm{n}}=\alpha_{1}$. These relations provide a link between the parameters of the density-dependent terms $\mathrm{g}_{\tau}^{1}$ and $\mathrm{g}_{\tau}^{3}$. Moreover, the parameterization (12) is consistent with the isospin dependence $\mathrm{f}_{\mathrm{s}}(\beta)=1-\mathrm{f}_{\mathrm{n}}(\beta)$ and $\mathrm{f}_{\mathrm{n}}(\beta)=\beta$ which is adopted in the present study. 


\section{LINKS BETWEEN PAIRING IN UNIFORM MATTER AND IN NUCLEI}

To understand the link between pairing in uniform matter and in nuclei, let us discuss in this section a local density approximation (LDA) for the pairing field $\Delta_{\mathrm{n}}(r)$, defined as

$$
\Delta_{\mathrm{n}}^{\mathrm{LDA}}(r) \equiv \Delta_{\mathrm{n}}^{\mathrm{unif}}\left(k_{\mathrm{Fn}}(r), x_{\mathrm{p}}(r)\right)
$$

where $\Delta_{\mathrm{n}}^{\text {unif }}\left(k_{\mathrm{Fn}}, x_{\mathrm{p}}\right)$ is the pairing gap in uniform matter calculated for a given Fermi momentum $k_{\mathrm{Fn}}$ and proton fraction $x_{\mathrm{p}}$. The LDA consists in replacing these variables by the local ones defined in finite nuclei. The local Fermi momentum $k_{\mathrm{Fn}}(r)$ and the local proton fraction $x_{\mathrm{p}}(r)$ are thus defined as,

$$
\begin{aligned}
k_{\mathrm{Fn}}(r) & =\left(3 \pi^{2} \rho_{\mathrm{n}}(r)\right)^{1 / 3}, \\
x_{\mathrm{p}}(r) & =\rho_{\mathrm{p}}(r) /\left(\rho_{\mathrm{n}}(r)+\rho_{\mathrm{p}}(r)\right) .
\end{aligned}
$$

The neutron and proton densities, $\rho_{\mathrm{n}}(r)$ and $\rho_{\mathrm{p}}(r)$, are given by the HFB calculation in finite nuclei. We represent $k_{\mathrm{Fn}}(r)$ and $x_{\mathrm{p}}(r)$ for two mid-shell nuclei, ${ }^{110} \mathrm{Sn}$ and ${ }^{150} \mathrm{Sn}$ in Fig. 8. At the surface of the nuclei, the proton fraction is decreasing faster in ${ }^{150} \mathrm{Sn}$ than in ${ }^{110} \mathrm{Sn}$ and the local Fermi momentum $k_{\mathrm{Fn}}(r)$ is slightly larger in ${ }^{150} \mathrm{Sn}$ than in ${ }^{110} \mathrm{Sn}$. Then, if pairing correlations are important at the surface, the pairing fields $\Delta_{\mathrm{n}}^{\mathrm{LDA}}(r)$ in the LDA should depend on the isospin properties of the pairing interaction. For these nuclei, the pairing fields in the LDA are shown in Fig. 9 for each of the pairing interactions IS+IV Bare, IS+IV Induced and IS Bare. To this end, we have calculated the solution of the BCS equations in asymmetric matter [16] and used it as the pairing gap in the
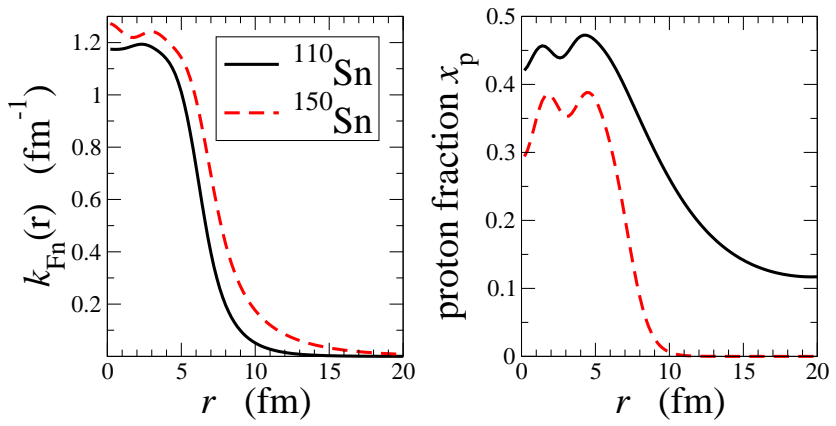

FIG. 8: (Color online) The neutron Fermi momentum and the proton fraction, obtained with the HFB densities, for two mid-shell Sn nuclei, ${ }^{110} \mathrm{Sn}$ (the solid line) and ${ }^{150} \mathrm{Sn}$ (the dashed line). 


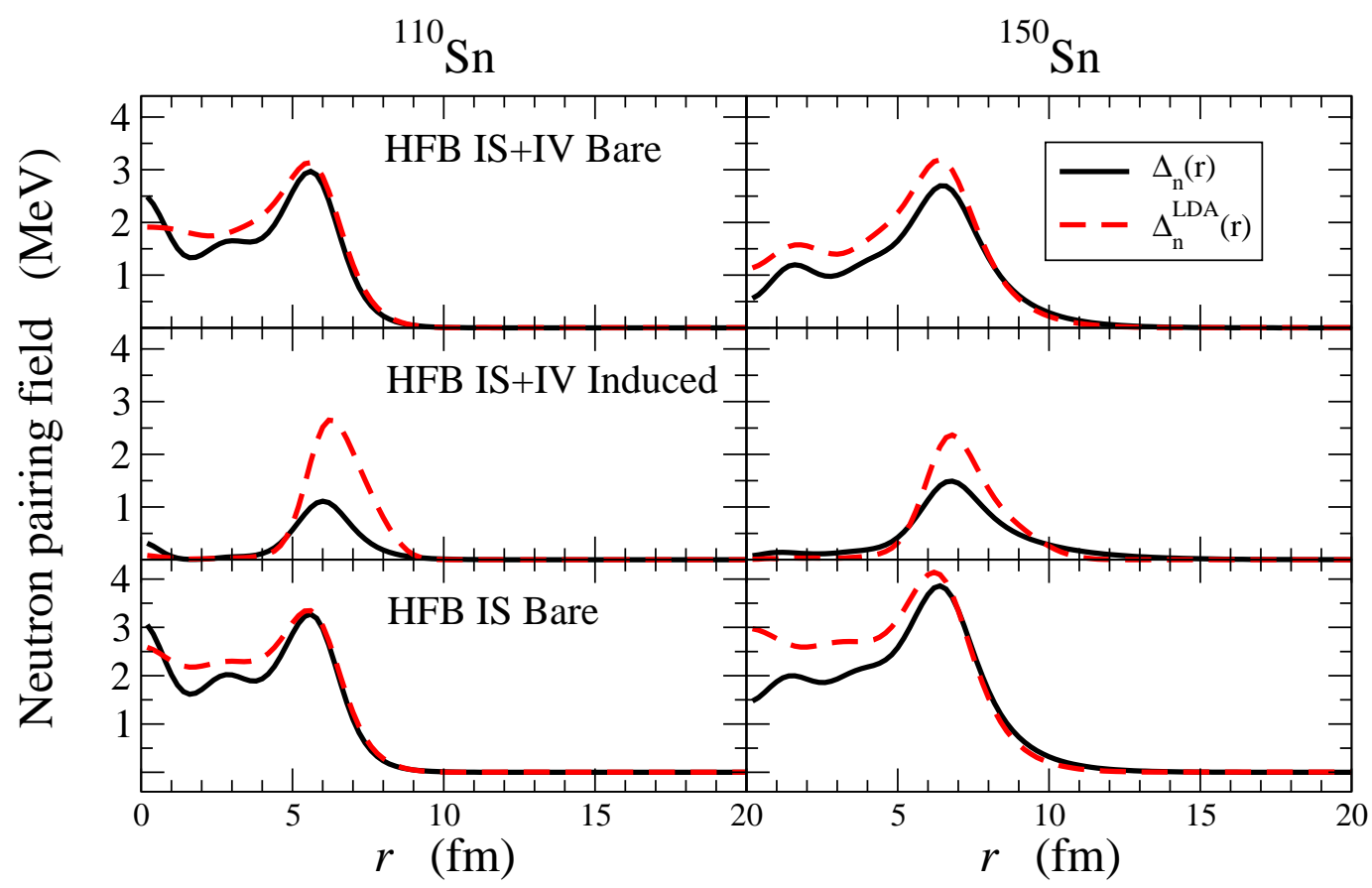

FIG. 9: (Color online) Comparison of the pairing field $\Delta_{\mathrm{n}}^{\mathrm{LDA}}(r)$ in the local density approximation (the dashed line) with that of the HFB calculations (the solid line) for the ${ }^{110} \mathrm{Sn}$ and ${ }^{150} \mathrm{Sn}$ nuclei. The pairing fields obtained with the different pairing interactions, IS+IV Bare, IS+IV Induced and IS Bare, are plotted separately.

uniform matter $\Delta_{\mathrm{n}}^{\text {unif }}\left(k_{\mathrm{Fn}}, x_{\mathrm{p}}\right)$, that is the same as the ones represented in Fig. 66. For a comparison, the pairing fields $\Delta_{\mathrm{n}}(r)$ obtained with the HFB calculations are also plotted in Fig. 9, It is clear from the figure that the pairing interactions IS+IV Bare and IS Bare have a mixed character of surface and volume types, while the pairing field obtained with the interaction IS+IV Induced is strongly surface peaked. It is surprising that the LDA provides not only qualitative but also quantitative description of the pairing field in finite nuclei. Nevertheless, finite size effects which are neglected in the LDA are not negligible, and the LDA overestimates the pairing field by about 10-20\% for the pairing interactions of the mixed volume and surface type correlations, like the IS+IV Bare and IS Bare ones, and by $50 \%$ for the pure surface type pairing correlations, like the IS+IV Induced interaction. From the pairing field, one could deduce the mean pairing gap according to Eq. (11). We show in Table \those mean pairing gaps obtained for ${ }^{150} \mathrm{Sn}$ for the set of pairing interactions IS+IV Bare, IS+IV Induced, and IS Bare. In addition, we also calculate the pairing gap 


\begin{tabular}{|c|c|c|c|c|c|}
\hline \multirow[t]{3}{*}{ nuclei } & \multirow{3}{*}{$\begin{array}{l}\text { pairing } \\
\text { interactions }\end{array}$} & \multicolumn{2}{|c|}{ HFB } & \multicolumn{2}{|c|}{ LDA } \\
\hline & & $\Delta_{\mathrm{n}}$ & $\tilde{\Delta}_{\mathrm{n}}$ & $\Delta_{\mathrm{n}}^{\mathrm{LDA}}$ & $\tilde{\Delta}_{\mathrm{n}}^{\mathrm{LDA}}$ \\
\hline & & $(\mathrm{MeV})$ & $(\mathrm{MeV})$ & $(\mathrm{MeV})$ & $(\mathrm{MeV})$ \\
\hline \multirow[t]{3}{*}{${ }^{110} \mathrm{Sn}$} & IS+IV Bare & 2.04 & 2.07 & 2.33 & 2.32 \\
\hline & IS+IV Induced & 0.39 & 0.57 & 0.68 & 1.19 \\
\hline & IS Bare & 2.32 & 2.32 & 2.60 & 2.53 \\
\hline \multirow[t]{3}{*}{${ }^{150} \mathrm{Sn}$} & IS+IV Bare & 1.72 & 1.71 & 2.16 & 2.04 \\
\hline & IS+IV Induced & 0.61 & 0.77 & 0.76 & 1.06 \\
\hline & IS Bare & 2.67 & 2.53 & 3.13 & 2.79 \\
\hline
\end{tabular}

TABLE II: The mean pairing gap $\Delta_{\mathrm{n}}$ and $\tilde{\Delta}_{\mathrm{n}}$ for ${ }^{110} \mathrm{Sn}$ and ${ }^{150} \mathrm{Sn}$ calculated with Eqs. (11) and (17), respectively. These are obtained by using either either the HFB pairing field $\Delta_{\mathrm{n}}(r)$ or the LDA pairing filed $\Delta_{\mathrm{n}}^{\mathrm{LDA}}(r)$ for the three density-dependent pairing interactions, IS+IV Bare, IS+IV Induced and IS Bare.

with another expression,

$$
\tilde{\Delta}_{\mathrm{n}} \equiv \frac{1}{\tilde{N}} \int d^{3} r \tilde{\rho}_{\mathrm{n}}(r) \Delta_{\mathrm{n}}(r)
$$

where $\tilde{N} \equiv \int d^{3} r \tilde{\rho}_{\mathrm{n}}(r)$ is the average number of neutrons participating to the pairing field. As expected, the average pairing gap is overestimated in the LDA approximation. For the interaction IS+IV Induced, the LDA even predicts a pairing gap smaller than the "experimental" one. It should also be remarked from the Table II that the average pairing gaps $\Delta_{\mathrm{n}}$ and $\tilde{\Delta}_{\mathrm{n}}$ are very similar for the surface and volume mixed-type pairing interactions IS+IV Bare and IS Bare, while there are important differences for the surface peaked interaction IS+IV Induced.

Notice that these LDA results are model dependent in a sense that they rely on a model for the neutron and proton density profile. However, except for this aspect, the local density 
approximation is qualitatively justified and the variation of the densities due to the pairing correlations are small. Presumably, one can consider that the LDA provides a reliable tool for a qualitative understanding of the pairing correlation in finite nuclei.

\section{CONCLUSIONS}

We have performed $\mathrm{HFB}$ calculations for semi-magic $\mathrm{Ca}, \mathrm{Ni}, \mathrm{Sn}$, and $\mathrm{Pb}$ isotopes and $N=20,28,50$ and 82 isotones using the density-dependent pairing interactions [16] deduced from microscopic nucleon-nucleon interaction [9]. Three interactions have been employed, namely, isospin dependent interactions adjusted to the pairing gaps both in symmetric and neutron matter obtained from the bare nucleon interaction (IS+IV Bare), or to those modified by medium polarization effects (IS+IV Induced), and an isoscalar interaction adjusted only in symmetric matter (IS Bare). We have compared the results of these pairing interactions with the experimental data for binding energies, two neutrons separation energies, and odd-even mass staggering (OES).

We have found that the two pairing interactions IS+IV Bare and IS+IV Induced lead to different results in finite nuclei. The comparison with the experimental OES suggests that the experimental data favor the interaction IS+IV Bare, which reproduces the bare pairing gap in both symmetric and neutron matter. These results indicate that the medium polarization effects estimated in infinite matter provides weaker pairing correlations than observed in finite nuclei. The discrepancies concerning the role of the phonon coupling between the calculations presented in Ref. [13, 14, 15] for finite nuclei and the calculations for uniform matter in Ref. [9], therefore, still remain an open question.

An interesting result shown in this paper is that the pairing interaction IS+IV Bare leads to good agreements with the experimental masses for light, intermediate and heavy nuclei without any tuning in different isotopes. This suggests that an inclusion of the isovector term in the effective pairing interaction helps in designing a global interaction applicable in the whole nuclear chart, taking advantage of the simplicity of the contact pairing interaction. It should however be noticed that in the proton pairing channel, the Coulomb interaction has not yet been included in our calculations. This should be done in futur investigations.

We have shown that the anomalous behavior of particle and pairing densities obtained in Ref. [11] for isosclar pairing interactions of surface type with the power of the density 
dependence $\alpha_{\mathrm{s}}<1 / 2$ is related to the large pairing gaps generated by these interactions at low density. The volume and surface mixed-type interaction adopted in the present study does not show this anomaly despite that the parameter $\alpha_{\mathrm{s}}$ is close to $1 / 2$.

Finally, we have discussed the local density approximation (LDA) for the pairing field, and have shown that it leads to a nice qualitative description of the pairing correlations in finite nuclei. The comparison of the pairing field obtained from the HFB calculation with the one extracted using the LDA suggests that there is a possibility to map from the pairing in uniform matter to that in finite nuclei.

\section{Acknowledgments}

One of us (J.M.) would like to thank M. Grasso for her help in the initial stage of this study. We also thank M. Matsuo, K. Matsuyanagi and W. Nazarewicz for usefull discussions. This work was supported by the Japanese Ministry of Education, Culture, Sports, Science and Technology by Grant-in-Aid for Scientific Research under the Program No. 19740115.

[1] J. Bardeen, L. N. Cooper, and J. R. Schrieffer, Phys. Rev. 108, 1175 (1957).

[2] A. Bohr, B. R. Mottelson, and D. Pines, Phys. Rev. 110, 936 (1958).

[3] D. M. Brink and R. Broglia, "Nuclear superfluidity, pairing in Finite Systems", Cambridge monographs on particle physics, nuclear physics and cosmology, vol 24 (2005).

[4] A. Schwenk, B. Friman, and G. E. Brown, Nucl. Phys. A713, 191 (2003).

[5] A. Fabrocini, S. Fantoni, A. Yu. Illarionov, and K. E. Schmidt, Phys. Rev. Lett. 95, 192501 (2005).

[6] T. Abe, and R. Seki, arXiv:nucl-th/0708.2523.

[7] A. Gezerlis, J. Carlon, arXiv:nucl-th/0711.3006.

[8] U. Lombardo, in "Nuclear Methods and the Nuclear Equation of State", Int. Rev. Nucl. Physics, Vol. 9, edited by M. Baldo (World Scientific, Singapore, 1999).

[9] L. G. Cao, U. Lombardo, and P. Schuck, Phys. Rev. C 74, 064301 (2006).

[10] D. J. Dean and M. Hjorth-Jensen, Rev. Mod. Phys. 75, 607 (2003).

[11] J. Dobaczewski, W. Nazarewicz, and P.-G. Reinhard, Nucl. Phys. A693, 361 (2001). 
[12] T. Duguet, Phys. Rev. C 69, 054317 (2004).

[13] F. Barranco, R. A. Broglia, G. Gori, E. Vigezzi, P.-F. Bortignon and J. Terasaki, Phys. Rev. Lett. 83, 2147 (1999).

[14] N. Giovanardi, F. Barranco, R. A. Broglia, and E. Vigezzi, Phys. Rev. C 65, 041304(R) (2002).

[15] F. Barranco, P. F. Bortignon, R. A. Broglia, G. Colò, P. Schuck, E. Vigezzi, and X. Viñas, Phys. Rev. C 72, 054314 (2005).

[16] J. Margueron, H. Sagawa, and K. Hagino, Phys. Rev. C 76, 064316 (2007).

[17] J. Dobaczewski, H. Flocard, and J. Treiner, Nucl. Phys. A422, 103 (1984).

[18] E. Chabanat et al, Nucl. Phys. A627, 710 (1997).

[19] M. Grasso, N. Sandulescu, N. Van Giai, and R. J. Liotta, Phys. Rev. C 64, 064321 (2001).

[20] M. Stoitsov, N. Michel, and K. Matsuyanagi, arXiv:nucl-th/0709.1006.

[21] G. F. Bertsch and H. Esbensen, Ann. Phys. (N.Y.) 209, 327 (1991).

[22] H. Esbensen, G. F. Bertsch and K. Hencken, Phys. Rev. C 56, 3054 (1997).

[23] E. Garrido, P. Sarriguren, E. Moya de Guerra, and P. Schuck, Phys. Rev. C 60, 064312 (1999).

[24] M. V. Stoitsov, J. Dobaczewski, W. Nazarewicz, S. Pittel, and D. J. Dean, Phys. Rev. C 68, $054312(2003)$.

See http://www.fuw.edu.pl/ dobaczew/thodri/thodri.html

[25] T. Duguet, P. Bonche, P.-H. Heenen, and J. Meyer, Phys. Rev. C 65, 014311 (2001).

[26] G. Audi, O. Bersillon, J. Blachot, and A. H. Wapstra, Nuclear Physics A729 (2003) 3; G. Audi, A. H. Wapstra, and C. Thibault, Nuclear Physics A729 (2003) 337.

[27] W. Satula, J. Dobaczewski, and W. Nazarewicz, Phys. Rev. Lett. 81, 3599 (1998).

[28] P. Vogel, B. Jonson, and P. G. Hansen, Phys. Lett. 139B, 227 (1984).

[29] S. Hilaire, J.-F. Berger, M. Girod, W. Stula, and P. Schuck, Phys. Lett. B 531, 61 (2002).

[30] M. Anguiano, J.L. Egido, and L.M. Robledo, Nucl. Phys. A 683, 227 (2001).

[31] J. Dobaczewski, W. Nazarewicz, and M. V. Stoitsov, Eur. Phys. J. A 15, 21 (2002). 